\title{
A Low-cost and Modular Receiver for MIMO SDR
}

\author{
Jo Verhaevert, Patrick Van Torre \\ INTEC-iMinds, Ghent University, Valentin Vaerwyckweg 1, B-9000 Gent, \\ Jo.Verhaevert@UGent.Be, Patrick.VanTorre@UGent.Be
}

\begin{abstract}
This article outlines the development of a low-cost receiver for Software Defined Radio (SDR) to receive a data burst on an intermediate frequency of $113 \mathrm{MHz}$. This data will have to be sampled and demodulated. Constellations are plotted in real time and Bit Error Rate (BER) is also continuously determined. The original data is received on $2.45 \mathrm{GHz}$ and transformed to an intermediate frequency by the receiver front-end. The received signal is then buffered, filtered, amplified and demodulated. This is all done in hardware. After this, the microcontroller takes over to process the signal, showing the data as a constellation diagram on the display.
\end{abstract}

Index Terms-Embedded Software Defined Radio, Demodulate, Digitize, Modular Embedded SDR.

\section{INTRODUCTION}

In this paper, the design and realization of a low-cost and modular receiver for Software Defined Radio (SDR) is described. All processes, including the channel information, run in real-time on a fast microcontroller, which results in a cheap solution. Employing channel feedback, the transmitter can then use this information to optimize its transmission. The modular aspect of this design implies that we have the possibility to interface multiple (identical) receivers to the microcontroller, resulting in a MIMO-system (Multiple Input Multiple Output).

This article will discuss in section II the realization of the complete hardware. The different hardware tests will be discussed in section III, followed by a description of the entire demodulation in section IV. Section V gives the conclusions.

\section{HARDWARE DESIGN}

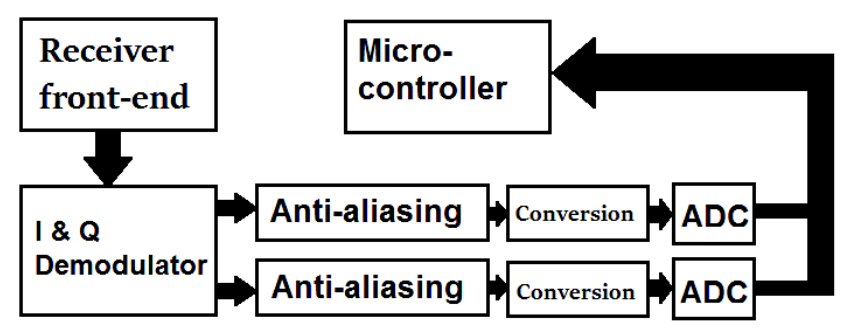

Fig. 1. Block diagram of the design.

The block diagram of the design is shown in Fig. 1. The received signal on $2.45 \mathrm{GHz}$ is processed by the receiver frontend and converted to a signal on $113 \mathrm{MHz}$. More information on this processing can be found in [1].
Initial analog signal processing is performed by the IQdemodulator, transforming the signal in baseband I and Q. The next block is the anti-aliasing circuit that is designed to block frequencies that are too high for the used sample rate. A last step is to digitize the signal and pass it on to the microcontroller for processing. The signal from the anti-aliasing filter has to be converted into a suitable form for the ADCs. This is due to the fact that the ADCs are using differential inputs and the signal coming from the IQdemodulator is a single-ended signal. The different signal processing blocks are now described in detail below.

\section{A. IQ-demodulator}

The IQ-demodulator is the JCIQ-176D from Mini-Circuits [2], a passive chip that demodulates the $113 \mathrm{MHz}$ signal using an LO (Local Oscillator), as further described. There are two possible ways to configure the LO frequency: $f_{L O}>f_{R F}$ or $f_{L O}<f_{R F}$. In the first case, $Q$ has a phase shift of $-90^{\circ}$ in comparison with $\mathrm{I}$, in the second case $90^{\circ}$. The maximum power on the LO and RF inputs is $50 \mathrm{~mW}$, corresponding to $16.99 \mathrm{dBm}$. The maximum output frequency of this chip is $5 \mathrm{MHz}$, limiting the bandwidth of the baseband signal.

\section{B. Low Pass Filter}

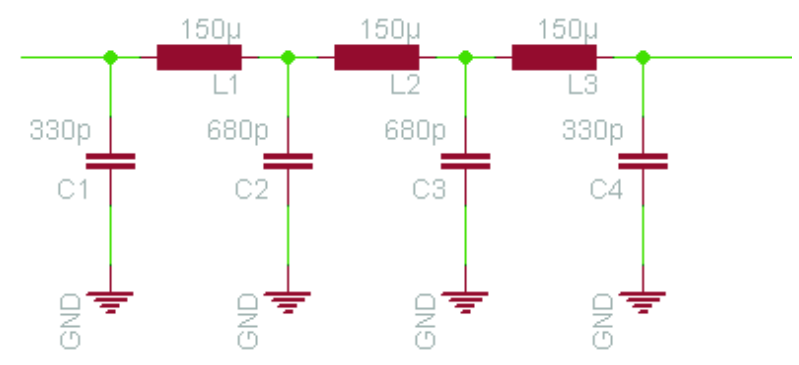

Fig. 2. Schematic diagram of the LC-Filter.

For this design, we need a steep LPF (Low Pass Filter), in order to prevent the occurrence of frequencies larger than $f_{\text {max }}$, which has been chosen to be smaller than the Nyquist frequency $f_{s}$. A Constant-K LPF has been chosen for its sharp slope, with a $f_{\text {cutoff }}$ of $1 \mathrm{MHz}$ and an impedance of $500 \Omega$. As shown in Fig. 2, all inductors in this configuration have equal values. The filter has been optimized to match the impedance of the single-ended to differential translator. Because of this higher impedance, a buffer is required between the demodulator and the filter. 


\section{Buffer}

The transistor BC848 has been chosen to be used as buffer [3]. This transistor in a common base configuration acts as a wide band buffer. The used circuit is shown in Fig. 3. $R_{2}$ is used to get a $50 \Omega$ input resistance. This is possible thanks to the parallel internal resistance of the transistor. $R_{1}$ and $R_{2}$ take care of the needed voltages at each point. $C_{1}$ and $C_{2}$ are coupling capacitors, $C_{3}$ is a decoupling capacitor.

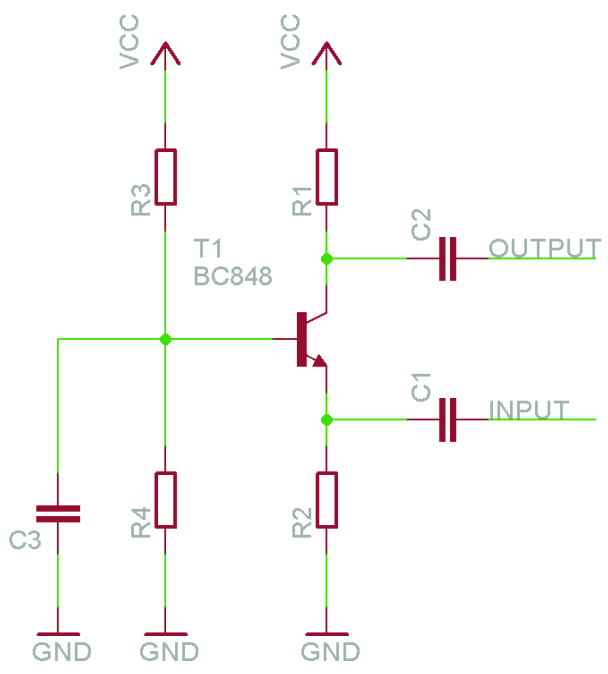

Fig. 3. Common base circuit of a transistor.

\section{Auto gain amplifier}

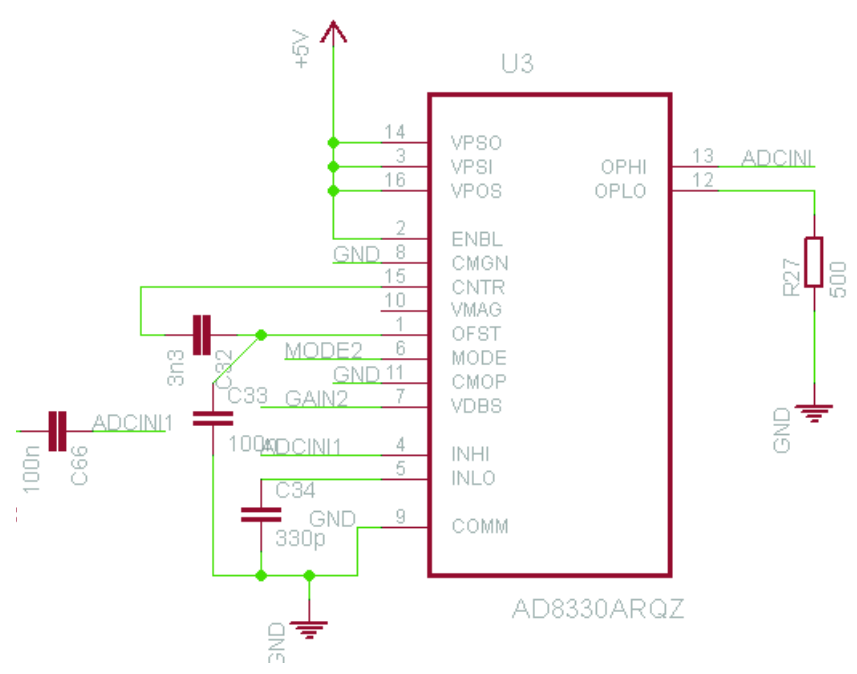

Fig. 4. Schematic diagram of the auto gain amplifier.

In order to ensure that the range of the ADC is optimally used, the signal coming from the demodulator must be amplified. For this purpose the AD8330 [4] is selected, a variable gain amplifier in which the gain can be adjusted by an analog signal output from the microcontroller. The amplifier has a bandwidth of $150 \mathrm{MHz}$, and can amplify signals of $0.3 \mathrm{mV}_{\mathrm{rms}}$ to $1 \mathrm{~V}_{r m s}$ at the input. In addition to this wide bandwidth amplifying capability, it has an adjustable gain from $0 \mathrm{~dB}$ to $50 \mathrm{~dB}$. The AD8330 is intended to amplify differential signals. It is however also possible to amplify single-ended signals as long as a few changes are being made to the circuit. The circuit is displayed in Fig. 4.

\section{E. $A D C$}

For the ADC an LTC1746 [5] is selected. The chip has a resolution of 14 bits, which is satisfactory for an SDR. Before the ADC can be connected to the AD8330, the signal must be converted from a single-ended to a differential signal. In order to realize this circuit the opamp LT1810 [6] with very high bandwidth and low distortion has been selected.

The ADC has a differential clock input (ENC/ENC). Provided that there is no differential clock available, there are three different ways to provide a single-ended clock to the ADC. A transformer, a single-ended clock with an offset voltage of $2 \mathrm{~V}$, or a CMOS-to-PECL translator can be used [7]. The translator has been chosen, providing a simple and reliable method. Apart from the need to convert the clock signal, the clock must be of a low jitter type with a minimum frequency of $1 \mathrm{MHz}$.

\section{F. Microcontroller}

The most important requirement for the microcontroller is the ability to read the data fast enough, and be able to process it. Except the speed requirement, it should also have enough IO-pins, at least $3 \times 16$ bit ports, since they are used by the ADCs. The presence of a graphic LCD display is also desirable.

The MCBSTM32F400 [8], [9] (including an LCD screen) was selected, featuring a high performance ARM CortexM4 processor and having extensive libraries available and with $8 \mathrm{MB}$ NOR Flash $512 \mathrm{MB}$ NAND flash and $2 \mathrm{MB}$ SRAM. This extra memory can be used for the storage of long sequences of data.

\section{G. LO-synthesizer}

The LO-synthesizer is the CDCE706 from Texas Instruments [10], this is a 3-PLL clock synthesizer that can be programmed using the $I^{2} \mathrm{C}$-bus [11]. The data can be stored in the EEPROM, hence it needs to be programmed only once. The $I^{2} C$ lines, SDA and SCLK, are connected to the supply voltage by means of pull-up resistors. Of the 6 possible outputs, only one is actively used. The other outputs are disabled in software. The input clock can be selected out of different clock sources, a crystal oscillator (XO), a differential clock (from XO or other source), or a simple crystal with associated capacitors. A clock signal of $16 \mathrm{MHz}$ is provided by a crystal which, together with the two capacitors and the internal circuitry of the CDCE706 forms an oscillator. The Phase Locked Loop (PLL) controls the frequency of the internal RF oscillator, employing reference and signal dividers. 


\section{HARDWARE TESTS}

\section{A. LO-synthesizer}

Fig. 5 shows the harmonic frequencies of the synthesizer. The first harmonic frequency (marker 2 in the figure) is approximately $10 \mathrm{~dB}$ below the fundamental frequency. Fig. 6 shows the fundamental frequency of the synthesizer and the spurious sidebands at least $44 \mathrm{~dB}$ below the carrier.

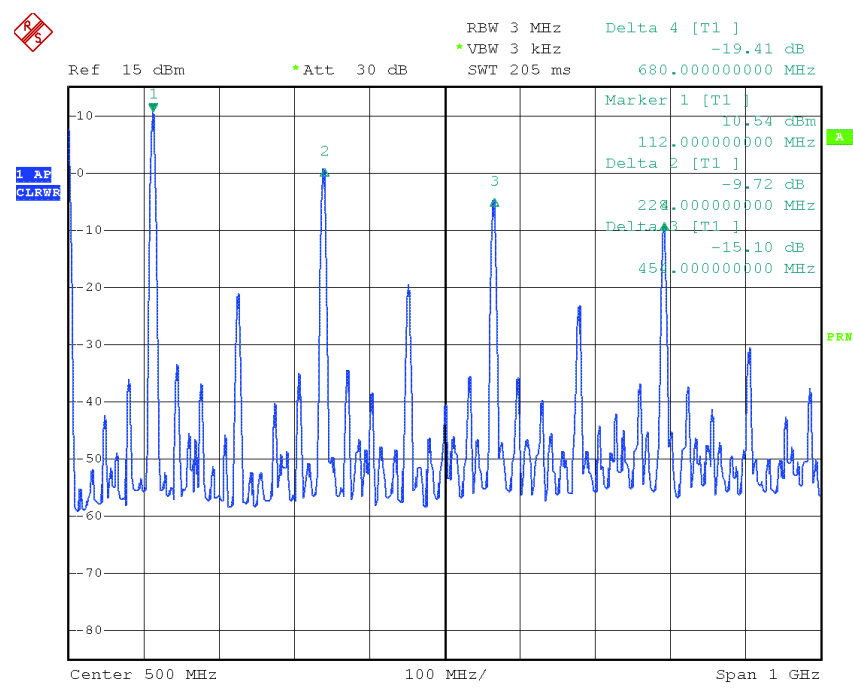

Fig. 5. Harmonic frequencies of LO-Synthesizer.

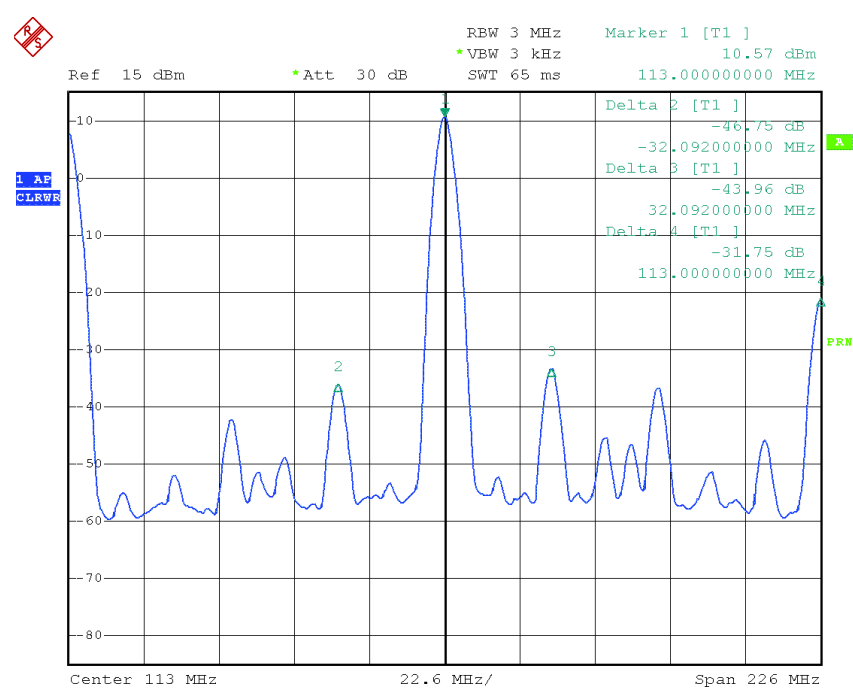

Fig. 6. $113 \mathrm{MHz}$ center frequency.

The oscilloscope image (Fig. 7) of the synthesizer output clearly shows a signal with limited over- and undershoot. From these measurements one can conclude that the LO-board works properly and can be used for the IQ-demodulator on the RFboard. The signal strength of $10.54 \mathrm{~dB}$ is within the range for the LO expected by the IQ-demodulator.

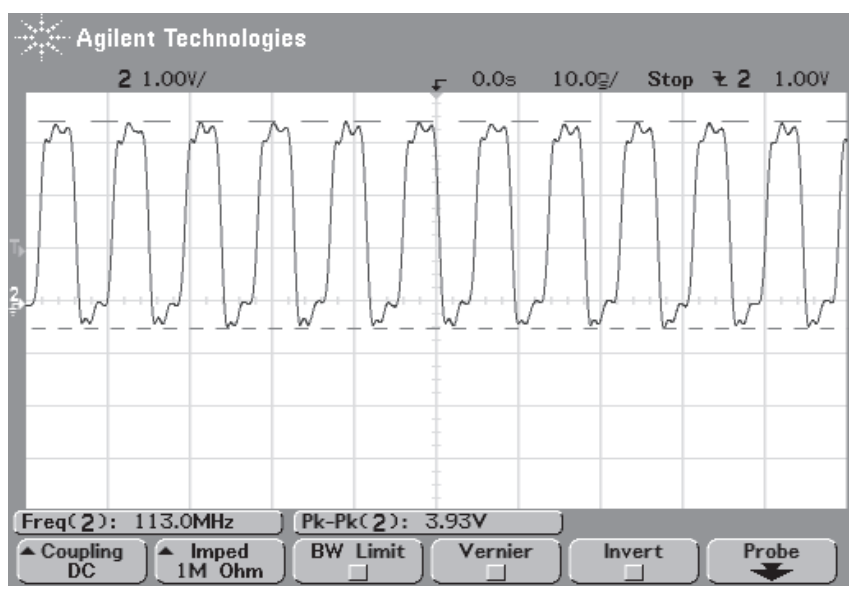

Fig. 7. LO-synthesizer signal.

\section{B. Buffer \& filter}

The cut-off frequency of the Constant-K filter is calculated at $1 \mathrm{MHz}$. Fig. 8 shows that signals between 1 and $2 \mathrm{MHz}$ begin to attenuate. The yellow line is the input of the filter and the blue line the output. Signals above $1.2 \mathrm{MHz}$ no longer pass the filter with a detectable voltage. In order to receive signals with a frequency above $f_{\text {cut }}$ (the cut-off frequency), the filter will have to be adjusted to one with a higher cut-off frequency and with the sample rate increased. In these figures, the phase shift is also clearly visible.
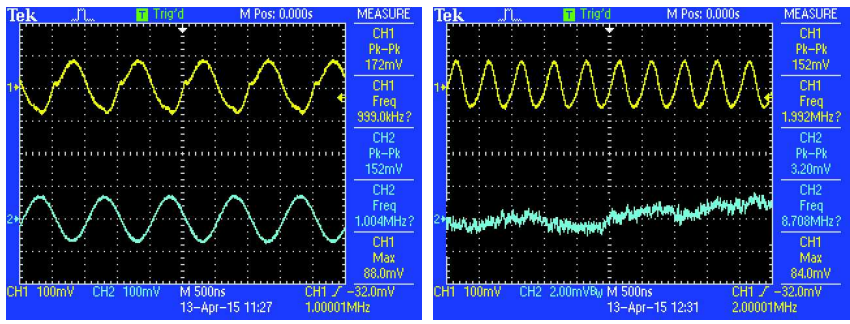

Fig. 8. Buffer/filter at $1 \mathrm{MHz}$ (left) and at $2 \mathrm{MHz}$ (right), $\mathrm{CH} 2$ vertical scale $100 \mathrm{mV} /$ div. and $2 \mathrm{mV} /$ div. respectively.

\section{Demodulation}

\section{A. Set-up}

The set-up for the demodulation consists of 4 boards, which need to be linked to each other in the correct way and fed with the appropriate voltages. The first board in the series is the receiver front-end and was realized in [1]. The demodulated signal is connected to the input of the receiver front-end. The mixed signal can then be offered to the RF-board. The receiver front-end has a third connector, this is the output of the onboard synthesizer. By using this output, the synthesizer can be set to the desired frequency by using a spectrum analyzer.

The converted signal coming from the receiver front-end is offered to the RF-board shown in Fig. 9. The input of the RFboard is located on the left side of the demodulator chip. This 


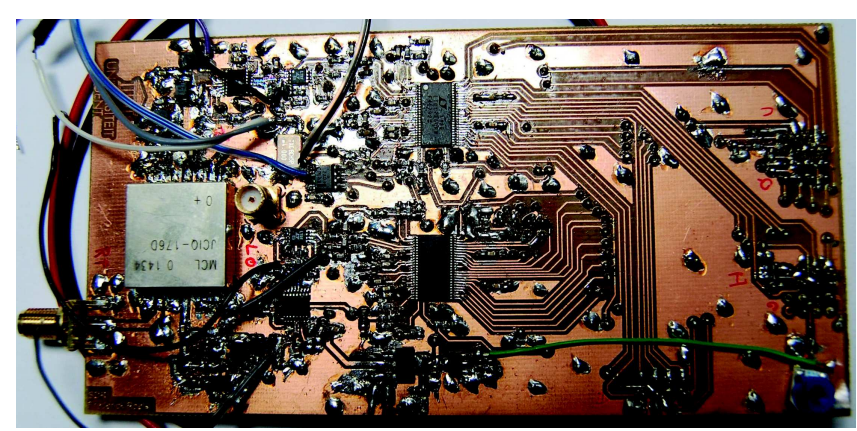

Fig. 9. RF-board.

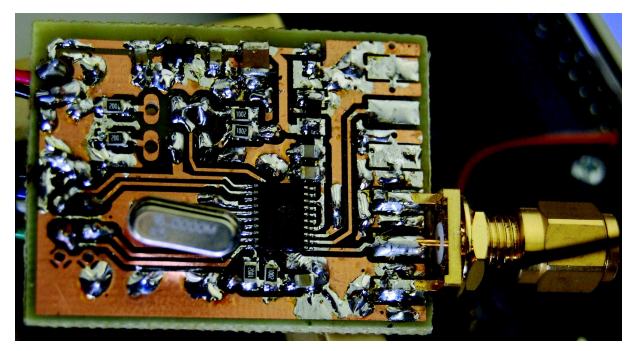

Fig. 10. Synthesizer board.

board has to be provided with an LO-signal, by means of the connector on the top side of the PCB. After processing, the data is offered to the microcontroller. The synthesizer board is shown in Fig. 10. The connector on the right side of the figure connects the synthesizer to the RF-board.

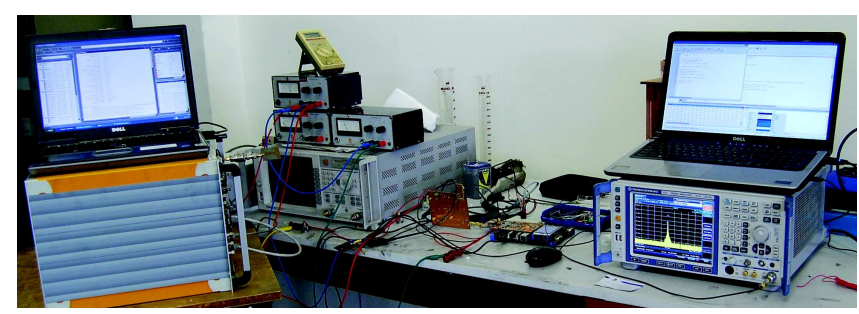

Fig. 11. Entire measurement set-up.

The entire measurement set-up is shown in Fig. 11. The transmitted 2.45 GHz signal is derived from the Signalion [12], controlled by Matlab. This can be seen on the left side of the figure. The spectrum analyzer is used to determine whether the receiver front-end, and the synthesizer are set-up correctly.

\section{B. Results}

After reading the data into the microcontroller, the data is logged by the computer using the debug interface. This data can then be used to carry out the processing in Matlab. After subtracting the mean value, we obtain the data represented in Fig. 12. There are three distinct parts. First, there is the gap which is clearly present, a second is an ASK modulated signal that serves as a pilot. Finally, there is the QPSK modulated data. As can be seen in the figure, part of the previous frame is also contained in the data read by the microcontroller. Since

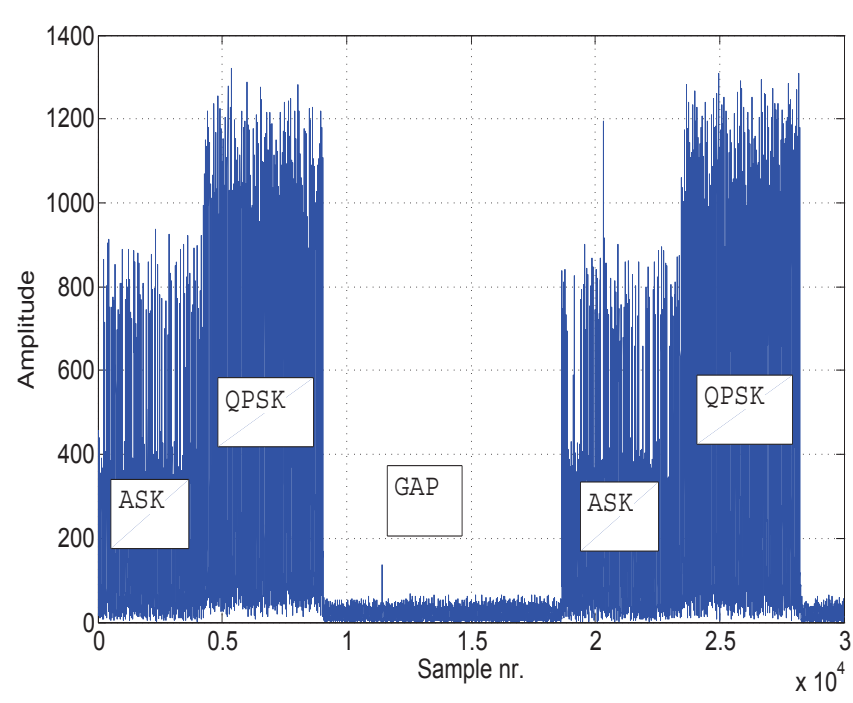

Fig. 12. Data frame with gap and ASK/QPSK modulation.

the start position of the signal within the data is not known, the end of the gap has to be found by the SDR processing [13].

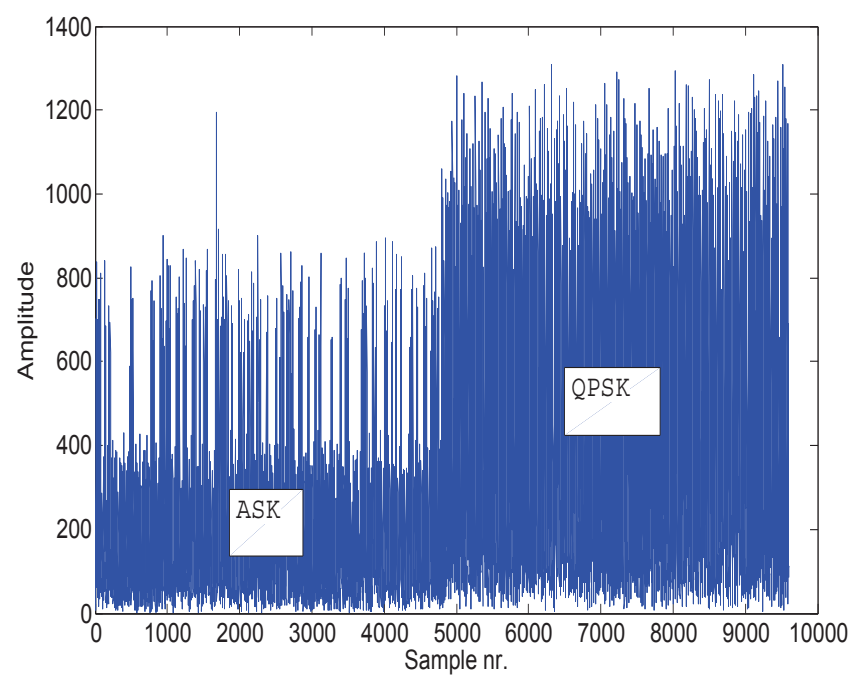

Fig. 13. Data frame.

After determining the position of the frame, it can be filtered out of the data in such way that only the necessary part remains. This frame, without the gap, can be found in Fig. 13, already time-synchronized coarsely. Next, this data frame can be processed using the Oerder \& Meyer algorithm (as described in [14]). This algorithm is used to determine the maximum value in the 32 samples of each symbol, providing fine time-synchronization, and then decimated. The values of the Oerder \& Meyer algorithm are graphically displayed in Fig. 14, in which a clear peak can be found. It is the sample position corresponding to this peak which will be used to decimate the data. 


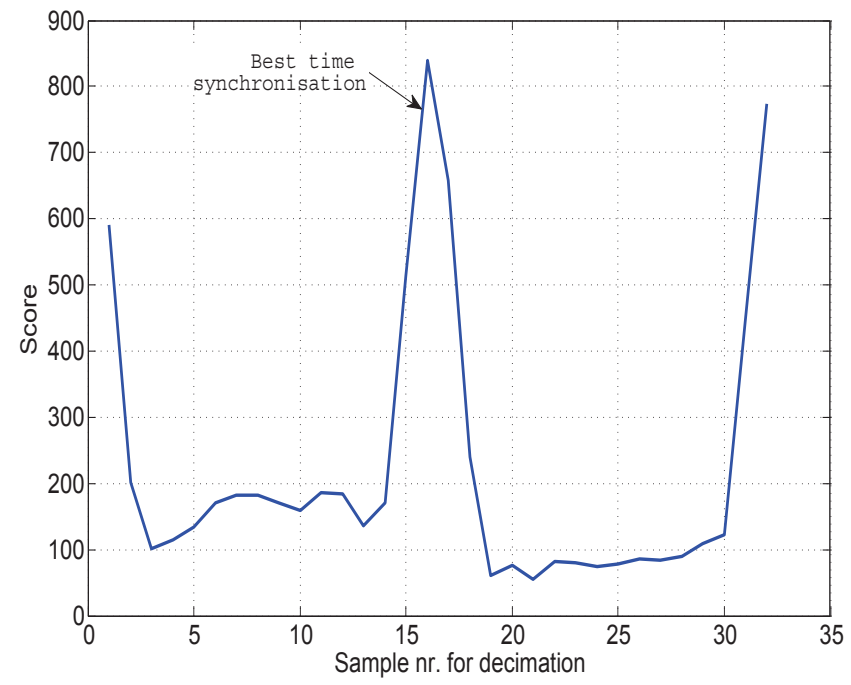

Fig. 14. Oerder \& Meyer algorithm.

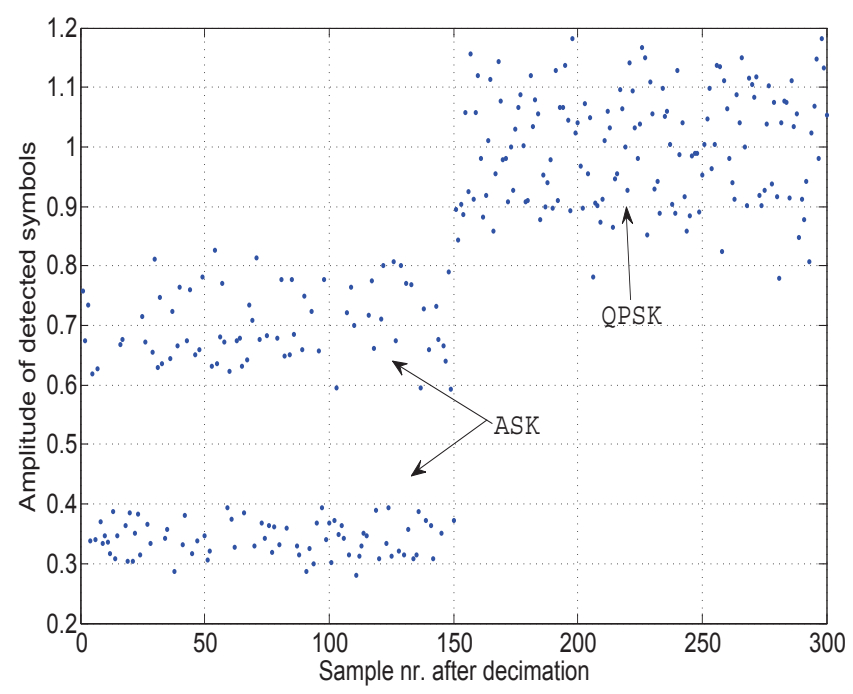

Fig. 15. Plot of ASK and QPSK.

After decimating, the table length is reduced from 9600 to 300 points. Fig. 15 displays these values graphically. The first 150 samples correspond to the signal modulated in ASK. The two levels used are clearly distinguished, indicating that the original data can be recovered. The last 150 samples correspond to the signal modulated in QPSK. After determining the offset frequency and the remaining phase offset, a constellation diagram is displayed, as shown in Fig. 16, illustrating that a perfect reception of QPSK signal is possible.

\section{Conclusions}

The first part includes the design of a PCB to demodulate and digitize the received signals. After making the PCB and soldering the components, the board could be tested step by step. The results obtained in section III conclude that the

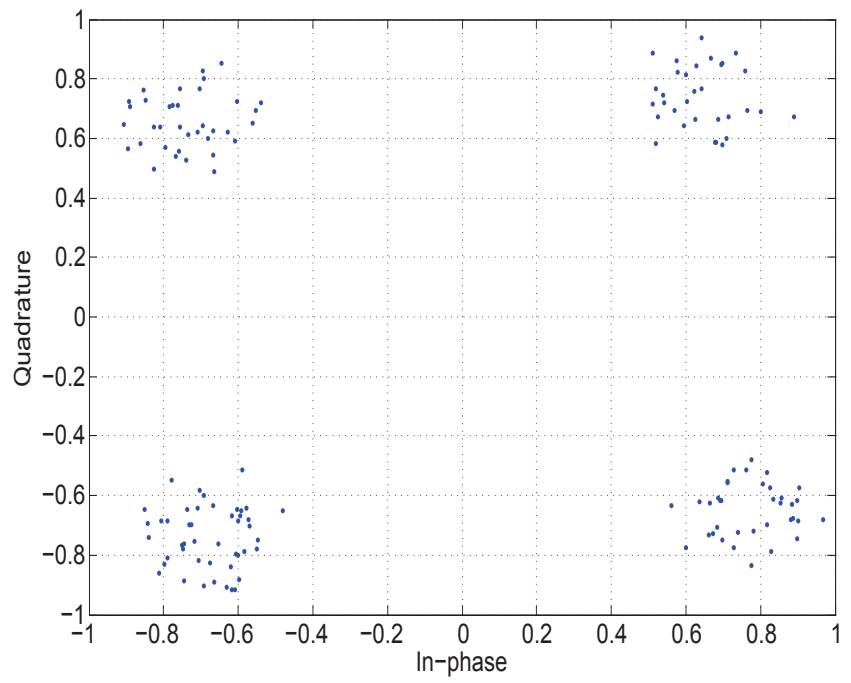

Fig. 16. Constellation diagram of QPSK part

hardware delivers the expected results. A possible further adjustment would be the integration of the synthesizer board resulting in a single $\mathrm{PCB}$ receiver.

\section{ACKNOWLEDGMENT}

The authors want to express their gratitude to Electronics Engineering student Frederick Roels for the fruitful cooperation and the interesting work. Without his efforts, the practical implementation would not have been realized.

\section{REFERENCES}

[1] J. Verhaevert and P. Van Torre, Design and Realization of a $2.45 \mathrm{GHz}$ Transmitter and Receiver as a Modular Unit for a MIMO SDR, 2015 Loughborough Antennas \& Propagation Conference (LAPC 2015), p. 28-31, Nov. 2015, Loughborough, United Kingdom.

[2] Mini-Circuits, IQ-demodulator, JCIQ-176D, Rev. B, 03/2015.

[3] NXP, BC848 series, BC848, Rev. 07, 11/2009.

[4] Analog Devices, Low Cost, DC to $150 \mathrm{MHz}$, Variable Gain Amplifier, AD8330, Rev. G, 03/2015.

[5] Linear Technology Corporation, Low Power, 14-bit, 25MSPS ADC, LTC1746, Rev. 3, 01/2015.

[6] Linear technology Corporation, Single/Dual $180 \mathrm{MHz}, 350 \mathrm{~V} / \mu \mathrm{s}$, Railto-Rail Input and Output, Low Distortion Opamps, LT1810, Rev. A, 03/2015.

[7] ON Semiconductor, 3.3V Dual LVTTL/LVCMOS to Differential LVPECL Translator, MC100LVELT22, Rev. 9 04/2012.

[8] STMicroelectronics, STM32f407, Rev. 4, 06/2013.

[9] Keil MCBSTM32F400, http://www.keil.com/mcbstm32f400/, Accessed: 2015-09-28

[10] Texas Instruments, Programmable 3-PLL Clock Synthesizer/Multiplier/ Divider, CDCE706, Rev. B, 11/200/.

[11] NXP Semiconductors Philips, I2C Manual, AN10216-01, http://www. nxp.com/documents/application_note/AN10216.pdf, 03/2003

[12] Signalion GmbH, HaLo 430 Hardware In The Loop MIMO Prototyping \& Monitoring Platform, Dresden.

[13] J. Verhaevert and P. Van Torre, Realization and MIMO-link measurements of a transmit module for spatial modulation, 9th European Conference on Antennas and Propagation (EuCAP2015), pp. 1-5, 1317 April 2015, Lisbon, Portugal.

[14] M. Oerder and H. Meyr, Digital Filter and Square Timing Recovery, IEEE Transactions on Communications, vol. 36, pp. 605-612, 1988. 